\section{Genomic and outcome analysis of adult T-cell lymphoblastic lymphoma}

T-cell lymphoblastic lymphoma (T-LBL) is a rare and aggressive lymphoid neoplasm occurring predominantly in children and young adults. ${ }^{1} \mathrm{~T}-\mathrm{LBL}$ is characterized by a proliferation of $\mathrm{T}$ lymphoblasts arrested at an early stage of maturation and accounts for less than $2 \%$ of all the non-Hodgkin lymphomas. Currently, the molecular pathogenetic mechanisms of T-LBL are largely unknown. Previous studies have identified recurring genetic alterations in NOTCH, PI3K/AKT and RAS signaling pathways in T-LBL patients; ${ }^{3-5}$ however, the genome-wide mutational landscape of T-LBL patients remains elusive. To address this question, we performed whole-exome sequencing in a cohort of 96 patients with T-LBL in the present study.

All patients (nine pediatric and 87 adult T-LBL patients) were reviewed and interpreted independently by three experienced pathologists. Diagnoses were made according to the current World Health Organization classification criteria. The clinical characteristics of the patient cohort are summarized in the Online Supplementary Table $S 1$, and the experimental design is depicted in the Online Supplementary Figure S1. The study was conducted in accordance with the Declaration of Helsinki and with the approval of the Institutional Review Board of the First Affiliated Hospital of Zhengzhou University. Wholeexome sequencing was performed using DNA extracted from 96 T-LBL patient tumor samples and 41 paired normal tissue samples. Detailed descriptions of wholeexome sequencing and bioinformatics analysis are provided in the Online Supplementary Materials and Methods. The 41 patients with paired normal tissue were deemed a "discovery cohort," and the remaining patients were considered a "validation cohort."

In the discovery cohort, the mean sequencing depth was $217 \times$, and a mean of $96.6 \%$ of the target sequence was covered to a depth of at least $50 \times$ after excluding the duplicates (Online Supplementary Table S2). A total of 1,599 nonsilent mutations (median 33, range 4-124) were identified (Online Supplementary Figure S2A and Table S3). We further evaluated the relationship between the somatic nonsilent mutation burden and the clinical features of T-LBL patients. The results showed that the somatic nonsilent mutation burden was associated with age $\left(\mathrm{R}^{2}=0.16, P=0.010\right.$; Online Supplementary Figure $\left.S 2 B\right)$ but not with other clinical features of these patients (sex, stage, or LDH). The predominant type of substitution was the $\mathrm{C}$ to $\mathrm{T}$ transition at NpCpG sites in T-LBL (Online Supplementary Figure S3A). Combined nonnegative matrix factorization clustering and correlation with the 30 curated mutational signatures defined by the Catalog of

Table 1. Characteristics of the 66 adult T-cell lymphoblastic lymphoma patients treated uniformly with Hyper-CVAD regimen according to their PHF6 or N/F mutation status.

\begin{tabular}{|c|c|c|c|c|c|c|c|}
\hline \multirow[b]{2}{*}{ Characteristics } & \multirow[b]{2}{*}{$\begin{array}{c}\text { Total } \\
(n=66)\end{array}$} & \multicolumn{2}{|c|}{ PHF6 } & \multicolumn{4}{|c|}{$N / F$} \\
\hline & & $\begin{array}{c}\text { wild-type } \\
(n=47)\end{array}$ & $\begin{array}{c}\text { mutation } \\
(n=19)\end{array}$ & $\mathbf{P}$ & $\begin{array}{c}\text { wild-type } \\
(n=39)\end{array}$ & $\begin{array}{c}\text { mutation } \\
(n=27)\end{array}$ & $P$ \\
\hline Age (years) & & & & 0.664 & & & 1.000 \\
\hline$\leq 50$ & 60 & 42 & 18 & & 35 & 25 & \\
\hline$>50$ & 6 & 5 & 1 & & 4 & 2 & \\
\hline Sex & & & & 0.304 & & & 1.000 \\
\hline Male & 54 & 40 & 14 & & 32 & 22 & \\
\hline Female & 12 & 7 & 5 & & 7 & 5 & \\
\hline Ann Arbor stage & & & & 0.203 & & & 0.078 \\
\hline $\mathrm{I} / \mathrm{II}$ & 16 & 9 & 7 & & 6 & 10 & \\
\hline III/IV & 50 & 38 & 12 & & 33 & 17 & \\
\hline LDH & & & & 0.176 & & & 0.211 \\
\hline Normal & 32 & 20 & 12 & & 16 & 16 & \\
\hline Elevated & 34 & 27 & 7 & & 23 & 11 & \\
\hline CNS involvement & & & & 0.316 & & & 0.138 \\
\hline No & 62 & 43 & 19 & & 35 & 27 & \\
\hline Yes & 4 & 4 & 0 & & 4 & 0 & \\
\hline BM involvement & & & & 0.496 & & & 0.757 \\
\hline No & 53 & 39 & 14 & & 32 & 21 & \\
\hline Yes & 13 & 8 & 5 & & 7 & 6 & \\
\hline Mediastinal involvement & & & & 0.311 & & & 0.393 \\
\hline No & 5 & 5 & 0 & & 2 & 3 & \\
\hline Yes & 61 & 42 & 19 & & 37 & 24 & \\
\hline Effusion & & & & 0.273 & & & 0.203 \\
\hline No & 27 & 17 & 10 & & 13 & 14 & \\
\hline Yes & 39 & 30 & 9 & & 26 & 13 & \\
\hline
\end{tabular}

T-LBL:T-cell lymphoblastic lymphoma; N/F: NOTCH1 and/or FBXW7;LDH: lactate dehydrogenase; CNS: central nervous system; BM: bone marrow,CVAD: cyclophosphamide, vincristine, doxorubicin in course A. 
Somatic Mutations in Cancer (COSMIC) database ${ }^{6}$ revealed three predominant signatures in T-LBL (Online Supplementary Figure $S 3 B$ ). The matched COSMIC signatures were signature 1 (signature A and C; cosine similarities, 0.94 and 0.93 , respectively) and signature 26 (signature B; cosine similarities, 0.88) (Online Supplementary Figure $S 3 C-D)$. Signature 1 is thought to result from the age-associated accumulation of 5-methylcytosine deamination events, while signature 26 is associated with defective DNA mismatch repair.

The mutational significance detection tool MuSiC was used to identify genes that were mutated at significantly higher rates than the background mutation rates, and 32 genes were identified with a false discovery rate value less than 0.1 (Online Supplementary Table S4). The spectrum and frequency of mutations in T-LBL in our study were similar to those of a previously published series of T-ALL. We also found several novel mutations in MTRNR2L2, CDC27, TMEM200C and NOTCH3, which have not been previously reported in T-LBL. To validate the potential driver mutations in the discovery cohort and to better define the gene-mutation prevalence in $\mathrm{T}$ LBL, we extended mutation detection to a validation cohort of 55 adults with T-LBL. In the validation cohort, all the common single-nucleotide polymorphisms (SNP) were filtered out except those identified as somatic mutations in our discovery cohort or in previous studies on $\mathrm{T}$ cell acute lymphoblastic leukemia (T-ALL). The accuracy of the genetic variant identification by exome sequencing was verified by performing Sanger sequencing in 140 variants from the same cases (Online Supplementary Table S5-6). We found that the Sanger sequencing data agreed with the exome sequencing data in $91 \%$ of the variants, confirming that our methods for exome sequencing and computational approaches for identifying genetic variants gave accurate results.

In total, NOTCH1 mutations were found in $35.6 \%$ (31 of 87) of adult patients with T-LBL (Figure 1 and Online Supplementary Table S7). These mutations were mainly clustered in the heterodimerization domain (HD) and/or

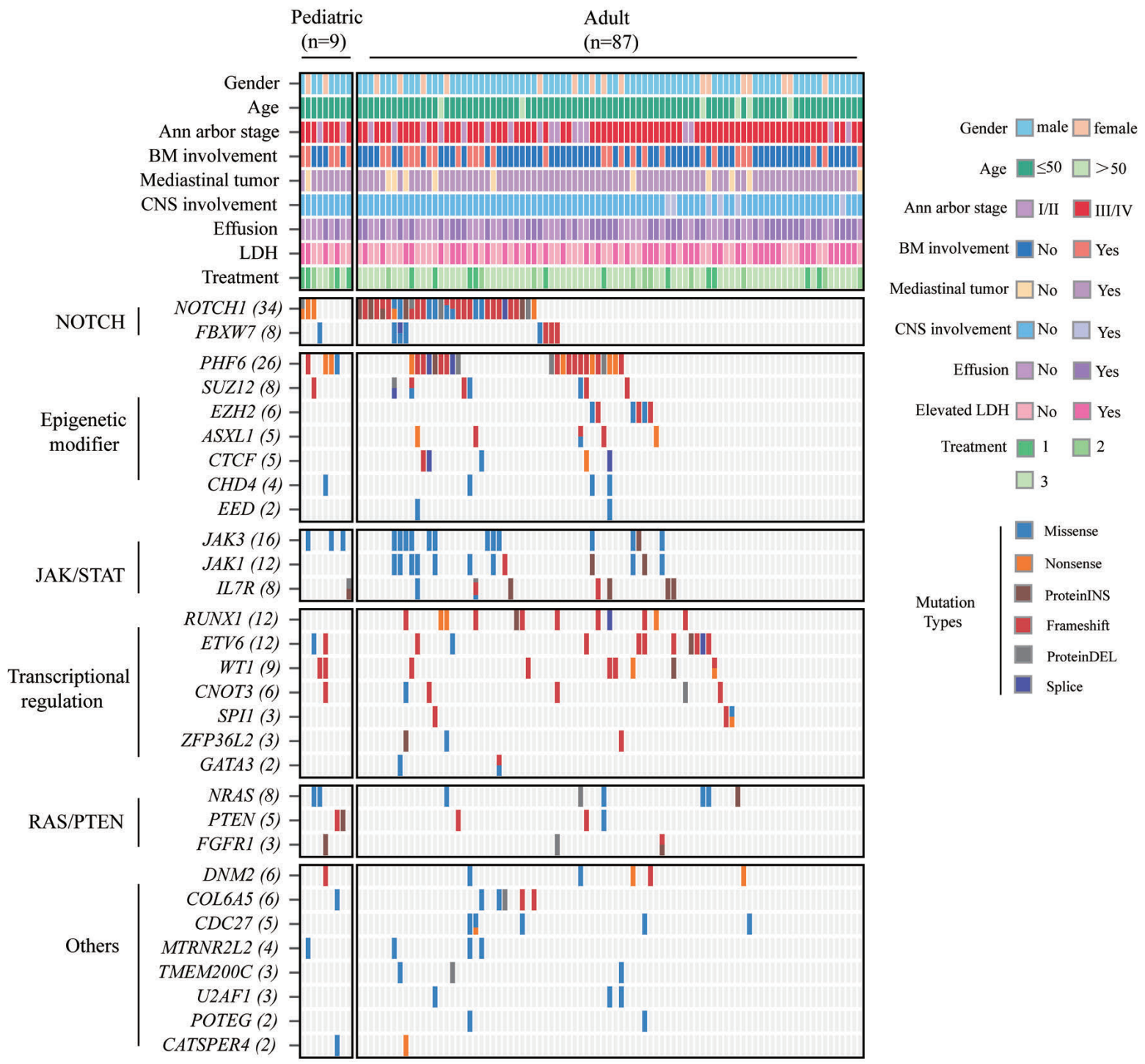

Figure 1. Mutational landscape of adult T-cell lymphoblastic lymphoma. The heat map shows the somatic mutational profile of the adult T-cell lymphoblastic lymphoma (T-LBL) cohort separated by gene functional groups. Rows represent individual genes, and columns represent individual tumors. Blocks are colorcoded by the functional type of mutation. The top panel shows the clinical characteristics of the T-LBL patient cohort. 
the proline, glutamine, serine and threonine (PEST) domain of NOTCH1 (Online Supplementary Figure S4). The HD domain mutations occurred predominantly in highly conserved amino acid residues, whereas the PEST domain mutations were all frameshifting indels or nonsense mutations and were therefore predicted to truncate the normal protein sequence. Similar mutations have been reported in T-ALL and activate NOTCH signaling. Mutations in $F B X W 7$, which functions as a negative regulator of NOTCH, were detected in $8.1 \%$ (7 of 87 ) of adult T-LBL cases. The majority of the mutations were identified in inactivating hotspots reported previously in T-ALL, including R465 ( $\mathrm{n}=2$ ), G423 and R505 (Online Supplementary Figure 4). In total, mutations in the NOTCH signaling pathway were identified in $40.2 \%$ (35 of 87) of adult patients with T-LBL.

PHF6 mutations were identified in 22 adult patients, representing the most frequently mutated epigenetic modifier in adult T-LBL (Figure 1). All mutations were truncating mutations (frameshift, nonsense or splice) and were expected to disrupt protein structure and function (Online Supplementary Figure S4). Other mutations were found in epigenetic modifiers, including $S U Z 12(\mathrm{n}=7)$, $E Z H 2(\mathrm{n}=6)$ and EED ( $\mathrm{n}=2)$, which encode the core component of Polycomb repressor complex 2 (PRC2) and mediate the repressive histone $\mathrm{H} 3$ lysine 27 trimethylation mark; CTCF $(\mathrm{n}=5)$, ASXL1 $(\mathrm{n}=5)$ and CHD4 $(\mathrm{n}=3)$, which are involved in chromatin organization, are also commonly found in adult patients with T-LBL (Figure 1). Altogether, we detected mutations in epigenetic modifiers in $36.8 \%$ (32 of 87 ) of the adult T-LBL cases.

We also found that recurrent mutations affected JAK/STAT signaling in $26.4 \%$ (23 of 87 ) of the cases, including JAK3 $(\mathrm{n}=13), J A K 1 \quad(\mathrm{n}=12)$ and $I L 7 R \quad(\mathrm{n}=7)$ (Figure 1). The majority of these mutations were detected in known activating hotspots or in close proximity, for example, JAK3 (M511, n=7; A573, n=2; R657, $\mathrm{n}=2$ and $\mathrm{A} 572, \mathrm{n}=1), J A K 1$ (R724, $\mathrm{n}=3)$ and IL7R (IL241-242, $\mathrm{n}=4$; VA253-254, $\mathrm{n}=2$; and V78, $\mathrm{n}=2$ ), which might result in constitutive activation of the JAK/STAT pathway (Online Supplementary Figure S4). Moreover, the mutation relation test revealed that $J A K 3$ was positively correlated with JAK1 $(P<0.01$, Online Supplementary Figure S5).

In addition, loss-of-function mutations in hematopoietic transcription factors, including ETV6 $(\mathrm{n}=10)$, RUNX1 $(\mathrm{n}=12)$, WT1 $(\mathrm{n}=7)$ and CNOT3 $(\mathrm{n}=5)$, and activating mutations in NRAS (G12 or G13, $\mathrm{n}=4$ ), were also frequently observed in patients with adult T-LBL (Figure 1), which was consistent with previous studies in T-ALL.

T-LBL is commonly treated with T-ALL-derived proto-

A
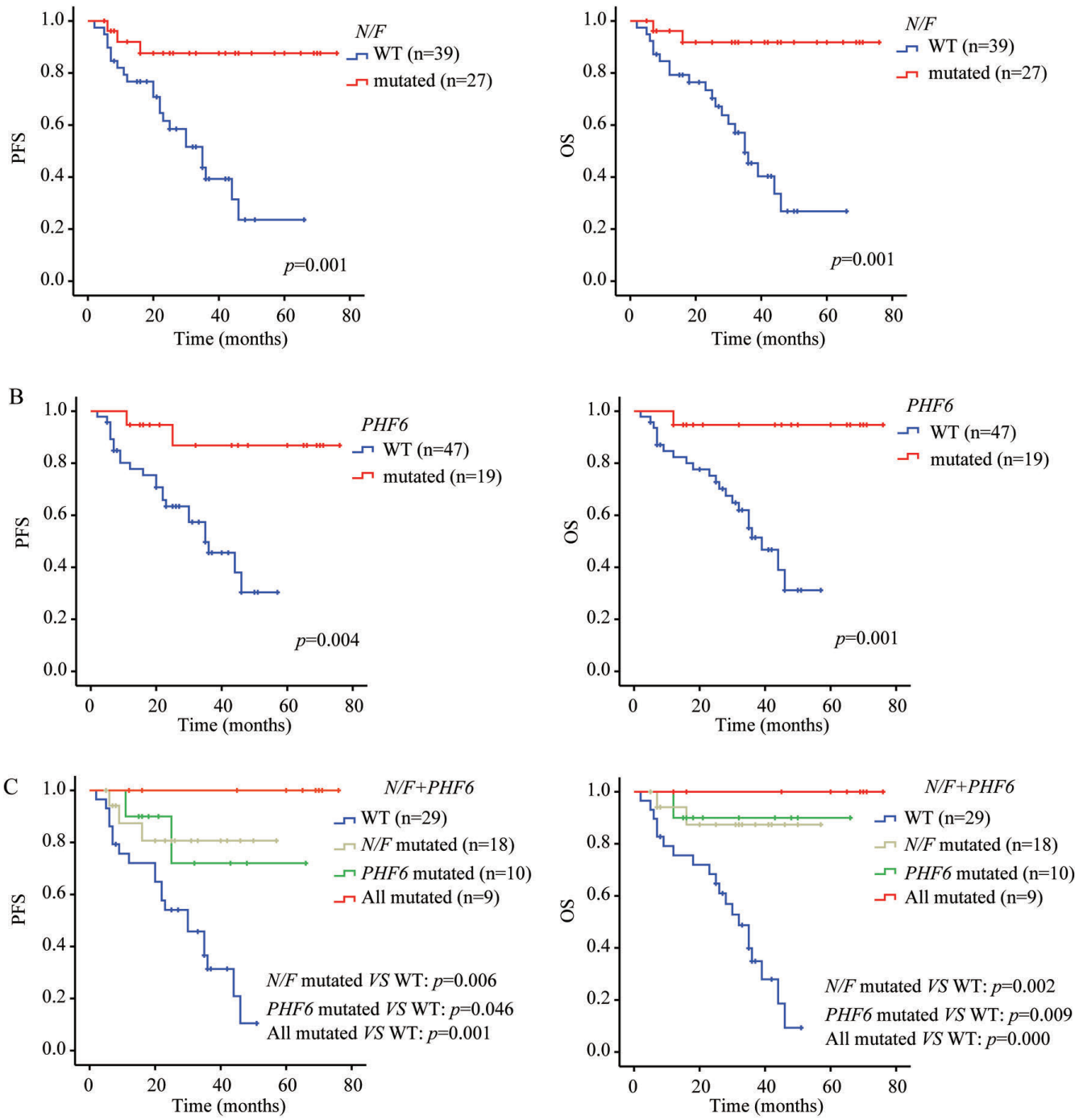

Figure 2. Mutational status and outcome analysis in adult T-cell lymphoblastic lymphoma patients who have been treated uniformly according to the hyper-CVAD regimen $(n=66)$. (A) PFS and OS in adult T-cell lymphoblastic lymphoma (T-LBL) patients according to $\mathrm{N} / \mathrm{F}$ mutationa status. (B) PFS and OS in adult T-LBL patients according to PHF6 mutational status. (C) PFS and OS in adult TLBL patients according to the combined genotype of N/F and PHF6. Survival curves were estimated using the Kaplan-Meier method and compared using a two-sided log-rank test. PFS: progression-free survival; OS: overall survival. 
cols. ${ }^{9}$ Therapeutic stratification based on prednisone response and minimal residual disease assessment is well established in T-ALL but not easy to extrapolate to TLBL. Molecular genetic markers are promising candidates for risk stratification because they represent underlying biological properties of the subgroups. ${ }^{10}$ Currently, molecular genetic markers such as NOTCH1 and/or FBXW7 (N/F) mutations, RAS/PTEN alterations, FLASH deletion, TCR status, and loss of heterozygosity at chromosome 6q, have been identified for risk stratification in pediatric T-LBL patients. To identify additional prognostic molecular markers in adult T-LBL, we further evaluated the prognostic value of the genetic mutations detected in our study. To avoid the potential bias caused by different therapeutic regimens, we analyzed 66 adult T-LBL patients who were treated uniformly according to the hyper-in course A: cyclophosphamide, vincristine, doxorubicin (CVAD) regimen14 (Table 1).

The univariate analysis showed that adult T-LBL patients with N/F mutations had improved overall survival (OS, $P=0.001$ ) and progression-free survival (PFS, $P=0.001)$ compared to those without the mutations (Figure 2A and Online Supplementary Table S8), which is consistent with previous reports. Of note, PHF6 mutations were significantly correlated with good prognosis in adult T-LBL patients. The estimated 3-year OS rates of patients with PHF6 mutations and those without PHF6 mutations were $94.7 \pm 5.1 \%$ and $51.4 \pm 8.4 \%$, respectively $(P=0.001$; Figure $2 \mathrm{~B})$. The estimated 3 -year PFS rates of the two groups of patients were $86.8 \pm 8.9 \%$ and $45.6 \pm 8.7 \%$, respectively $(P=0.004$; Figure $2 \mathrm{~B})$.

Moreover, Cox multivariate regression analysis demonstrated that $N / F$ and PHF6 mutation status were independent favorable prognostic markers in adult T-LBL after adjusting for multiple potential confounding clinical factors (age, Ann Arbor stage, bone marrow involvement, central nervous system involvement and $\mathrm{LDH}$ level) (Online Supplementary Table S8). Importantly, we found that adult T-LBL patients without N/F or PHF6 mutations had a much worse prognosis than individuals with mutations in the three genes, as reflected by the OS time and the PFS time (Figure 2C). Therefore, the N/F and PHF6 mutational status in adult T-LBL might provide an alternative for therapeutic stratification. However, these findings need to be further verified in large independent cohorts.

In summary, we provided the first comprehensive portrait of the mutational landscape of adult T-LBL. PHF6 mutational status may provide a novel marker of good prognosis in adult T-LBL.

Zhaoming Li, 1,2, Yue Song, ${ }^{1,2, \#}$ Yanjie Zhang, 1,3,\# Chaoping Li, ${ }^{1,3}$ Yingiun Wang, ${ }^{1,3}$ Weili Xue, ${ }^{1,3}$ Lisha Lu, ${ }^{1,3}$ Mengyuan Jin, ${ }^{1,3}$ Zhiyuan Zhou, ${ }^{1,3}$ Xinhua Wang, ${ }^{1,2}$ Ling Li, Lei Zhang, ${ }^{1,2}$ Xin Li, $i^{1,2}$ Xiaorui Fu, ${ }^{1,2}$ Zhenchang Sun, ${ }^{1,2}$ Jingjing Wu, ${ }^{1,2}$ Xudong Zhang, ${ }^{1,2}$ Hui Yu, ${ }^{1,2}$ Feifei Nan, ${ }^{1,2}$ $Y u$ Chang, 1,2 Jiaqin Yan, ${ }^{1,2}$ Xiaoyan Feng, ${ }^{1,2}$ Xiaolong Wu, ${ }^{1,2}$ Guannan Wang, ${ }^{4}$ Dandan Zhang, ${ }^{4}$ Wencai Li, ${ }^{4}$ Feixiang Li, Yuan Zhang, ${ }^{6}$ Ken H. Young ${ }^{7}$ and Mingzhi Zhang ${ }^{1,2,}$

"ZL, YS and YZ are co-first authors.

'Department of Oncology, The First Affiliated Hospital of Zhengzhou University, Zhengzhou, China; ${ }^{2}$ Lymphoma Diagnosis and Treatment Center of Henan Province, Zhengzhou, China; ${ }^{3}$ Institute of Clinical Medicine, The First Affiliated Hospital of Zhengzhou University, Zhengzhou, China; ${ }^{4}$ Department of Pathology, The First Affiliated Hospital of Zhengzhou University, Zhengzhou, China; ${ }^{5}$ Novogene Bioinformatics Technology Co., Ltd., Xueqing Road,
Beijing, China; ${ }^{6}$ The Academy of Medical Science of Zhengzhou University, Zhengzhou, China and 'Department of Hematopathology, The University of Texas MD Anderson Cancer Center, Houston, TX, USA

Funding: this study was supported by funds from the National Natural Science Foundation of China (81570203), Innovation Funds Project of the First Affiliated Hospital of Zhengzhou University (to Mingzhi Zhang), the scientific and technological project from health and Family Planning Commission (201702047) and Department of Science, Technology of Henan province (182102310114).

Acknowledgments: the authors gratefully acknowledge Dr. Tian Tian for critical review and language editing of the manuscript.

Correspondence: MINGZHI ZHANG.

mingzhi_zhang1@163.com

doi:10.3324/haematol.2019.220863

Information on authorship, contributions, and financial \& other disclosures was provided by the authors and is available with the online version of this article at wWw. haematologica.org.

\section{References}

1. Cortelazzo S, Ferreri A, Hoelzer D, Ponzoni M. Lymphoblastic lymphoma. Criti Rev Oncol Hematol. 2017;113:304-317.

2. Lepretre S, Graux C, Touzart A, Macintyre E, Boissel N. Adult T-type lymphoblastic lymphoma: Treatment advances and prognostic indicators. Exp Hematol. 2017;51:7-16.

3. Bonn BR, Huge A, Rohde M, et al. Whole exome sequencing hints at a unique mutational profile of paediatric T-cell lymphoblastic lymphoma. Br J Haematol. 2015;168(2):308-313.

4. Callens $\mathrm{C}$, Baleydier F, Lengline E, et al. Clinical impact of NOTCH1 and/or FBXW7 mutations, FLASH deletion, and TCR status in pediatric T-cell lymphoblastic lymphoma. J Clin Oncol. 2012; 30(16):1966-1973

5. Balbach ST, Makarova O, Bonn BR, et al. Proposal of a genetic classifier for risk group stratification in pediatric T-cell lymphoblastic lymphoma reveals differences from adult T-cell lymphoblastic leukemia. Leukemia. 2016;30(4):970-973.

6. Tate JG, Bamford S, Jubb HC, et al. COSMIC: the Catalogue Of Somatic Mutations In Cancer. Nucleic Acids Res. 2019;47(D1):D941D94

7. Liu Y, Easton J, Shao Y, et al. The genomic landscape of pediatric and young adult T-lineage acute lymphoblastic leukemia. Nat Genet. 2017;49(8):1211-1218

8. Seki M, Kimura S, Isobe T, et al. Recurrent SPI1 (PU.1) fusions in high-risk pediatric T cell acute lymphoblastic leukemia. Nat Genet. 2017;49(8):1274-1281

9. Uyttebroeck A, Suciu S, Laureys G, et al. Treatment of childhood Tcell lymphoblastic lymphoma according to the strategy for acute lymphoblastic leukaemia, without radiotherapy: long term results of the EORTC CLG 58881 trial. Eur J Cancer. 2008;44(6):840-846.

10. Marks DI, Rowntree C. Management of adults with T-cell lymphoblastic leukemia. Blood. 2017;129(9):1134-1142.

11. Lepretre S, Touzart A, Vermeulin T, et al. Pediatric-like acute lymphoblastic leukemia therapy in adults with lymphoblastic lymphoma: the GRAALL-LYSA LL03 Study. J Clin Oncol. 2016; 34(6):572-580

12. Trinquand A, Tanguy-Schmidt A, Ben Abdelali R, et al. Toward a NOTCH1/FBXW7/RAS/PTEN-based oncogenetic risk classification of adult T-cell acute lymphoblastic leukemia: a Group for Research in adult acute lymphoblastic leukemia study. J Clin Oncol. 2013; 31(34):4333-4342

13. Gutierrez A, Dahlberg SE, Neuberg DS, et al. Absence of biallelic TCRgamma deletion predicts early treatment failure in pediatric $\mathrm{T}$ cell acute lymphoblastic leukemia. J Clin Oncol. 2010;28(24):38163823 .

14. Thomas DA, O'Brien S, Cortes J, et al. Outcome with the hyperCVAD regimens in lymphoblastic lymphoma. Blood. 2004; 104(6):1624-1630

15. Jenkinson S, Koo K, Mansour MR, et al. Impact of NOTCH1/FBXW7 mutations on outcome in pediatric T-cell acute lymphoblastic leukemia patients treated on the MRC UKALL 2003 trial. Leukemia. 2013;27(1):41-47. 